\title{
Aspectos psicoeducativos en la evaluación del alumnado con Altas Capacidades Intelectuales: análisis de un caso
}

\section{Psychoeducational aspects in the assessment of students with High Intel- lectual Abilities: a single-case study}

\author{
Diego Jesús Luque-Parra* \\ Universidad de Málaga, España \\ Ricardo Hernández-Díaz \\ Junta de Andalucía, España \\ María Jesús Luque-Rojas \\ Universidad de Málaga, España \\ (Rec.: septiembre de 2015 - Acept.: marzo de 2016)
}

\section{Resumen}

El presente trabajo trata de favorecer la reflexión, desde la psicología educacional, sobre la respuesta a las necesidades específicas de apoyo educativo de los alumnos y alumnas con Altas Capacidades Intelectuales. Tras una breve exposición sobre las características de este tipo de alumnado, se realiza un análisis de caso con el que establecer la relación entre la evaluación psicoeducativa y la intervención psicopedagógica. Desde la labor de los psicólogos de la educación (interviniendo en todos los procesos psicológicos que afectan al aprendizaje o que se derivan de este) y otros colegas que actúan en el ámbito educativo, se buscaría, en última instancia, relacionar los objetivos de cualquier intervención psicoeducativa: la valoración diagnóstica y la planificación de la intervención pedagógica dentro de la atención a la diversidad desarrollada en el sistema educativo español.

Palabras clave: Altas Capacidades Intelectuales, necesidades específicas de apoyo educativo, intervención psicopedagógica, inclusión educativa.

\begin{abstract}
The following paper aims to promote an educational psychology-based reflection on the response to those specific educational needs required by students with high intellectual abilities. After a brief discussion on the characterization of this kind of student, a single-case study is developed in order to establish a relationship between psycho-educational assessments and educational psychology interventions. The work of educational psychologists (intervening in all the psychological processes affecting learning or resulted therefrom) and other acting colleagues in the educational field would ultimately seek to relate the objectives of any psychoeducational intervention: diagnostic assessment and educational intervention planning within the diversity emphasis developed on the Spanish educational system.
\end{abstract}

Keywords: high intellectual abilities, specific educational needs, educational psychology intervention, educational inclusion.

\footnotetext{
* Correspondencia a: Diego Jesús Luque-Parra. Departamento de Psicología Evolutiva y de la educación. Dirección postal: Universidad de Málaga. Campus de Teatinos, (29071) Málaga. E-mail: dluque@uma.es
} 


\section{Introducción}

Uno de los grandes retos a los que se enfrenta la educación durante el siglo XXI es la atención a la diversidad del alumnado desde la equidad y la inclusión, atención que se ha convertido en uno de los ejes esenciales donde se mueve la práctica docente. En efecto, el alumnado con discapacidades, los alumnos con problemas de aprendizaje y los más desfavorecidos social y culturalmente, han estado centrando la respuesta educativa como elemento compensador de sus dificultades. Sin embargo, hay también otro grupo de alumnos y alumnas situados, desde una exclusiva visión estadística, en el extremo derecho de la campana de Gauss, que necesita también una atención educativa para desarrollar de manera adecuada sus potencialidades (Torrego, 2011).

En España, la planificación de las actuaciones educativas se establece desde la Atención a la Diversidad del alumnado, basándose en una estructura de valoración educativa dirigida hacia una intervención psicopedagógica en las concreciones de los casos del alumnado. Así, el término de necesidades específicas de apoyo educativo hace referencia a los alumnos y alumnas que requieran una atención educativa diferente a la ordinaria por diversos motivos, tanto académicos o de aprendizaje como de discapacidad, altas capacidades intelectuales o cualesquiera otras condiciones personales o de historia escolar, persiguiendo alcanzar los objetivos de máximo desarrollo personal, intelectual, social y emocional en todos sus alumnos. Una atención que deberá tener un carácter integral, iniciándose desde el mismo momento en que dicha necesidad sea identificada y rigiéndose por los principios de normalización e inclusión.

De acuerdo a las precisiones anteriores, la atención a los alumnos y alumnas con altas capacidades intelectuales asume como principios de intervención:

- Disponer de los medios necesarios para alcanzar el máximo desarrollo personal, intelectual, social y emocional, así como los objetivos establecidos con carácter general.

- Establecimiento de procedimientos y recursos precisos, para la identificación temprana de las necesidades educativas específicas, así como su respuesta desde esa identificación.

- Desarrollo educativo regido por los principios de normalización e inclusión.

- Organización escolar y ajuste a través de las adecuaciones curriculares precisas para facilitar la consecución de los fines establecidos.

- Promoción de la formación del profesorado, profesionales, madres y padres para la mejor intervención educativa.
Cualquier planteamiento educativo dirigido al alumnado con Altas Capacidades Intelectuales (AACCII en adelante) debe contemplarse desde una perspectiva amplia, hacia un colectivo no homogéneo, aunque tengan en común valoraciones de altas capacidades. Ciertamente, el término AACCII va a hacer referencia a la coincidencia de posiciones en el extremo superior de la curva de distribución de la inteligencia, a lo que habrá de añadir otros elementos de evaluación. En la actualidad, aunque los expertos y los psicólogos educativos tienen dificultades para llegar a un acuerdo en la definición del concepto de AACCII (Arocas, Martínez \& Regadera, 2002; Fernández \& Perales, 2013), la inteligencia y su evaluación de capacidad y aptitudes ha sido el elemento central para poder comenzar a hablar de las altas capacidades.

De acuerdo con lo anterior, esta evaluación no solo vendrá definida por la inteligencia como criterio estadístico-psicométrico (Luque, 2014), sino que debe ir acompañada de un análisis de la creatividad, de los aspectos sociales, emocionales, de personalidad, así como de la valoración de la ejecución y rendimiento en los distintos procesos de enseñanza - aprendizaje para la adecuada definición y valoración diagnóstica que pudiera proceder.

En consecuencia, las AACCII supondrán la manifestación de un estado particular de funcionamiento cognitivo (alto nivel aptitudinal y de habilidades) caracterizado por potencialidades, facilidades de desarrollo o un gran número de estrategias y habilidades que presentan y utilizan. Desde esta apreciación, no obstante, se debe reconocer que estos alumnos y alumnas presentan necesidades específicas de apoyo educativo tanto a nivel cognitivo como afectivo, social y curricular, lo que les hace merecedores de una respuesta educativa y social ajustada.

Desde este marco, el presente trabajo pretende favorecer la reflexión en los psicólogos de la educación (interviniendo en todos los procesos psicológicos que afectan al aprendizaje, o que se derivan de este) y otros colegas de actuación en el ámbito educativo, sobre el alumnado con AACCII. Ello sin perder de vista el objetivo de cualquier intervención psicoeducativa: la valoración diagnóstica y la planificación de la intervención pedagógica, como respuesta a las necesidades educativas que presenta este alumnado.

\section{Aspectos definitorios}

No es fácil dar una definición única del término de AACCII, ya que el término ha estado, y está, muy unido al concepto de inteligencia y su medida. No existiendo además, una definición asumida en común por los especialistas en el tema. Desde este punto de partida, definir las AACCII vendrá dado, en parte, por la conceptuación de la inteligencia que realicemos. Así, la inteligencia ha sido definida de múltiples maneras 
(Calero, García \& Gómez, 2007), y se ha puesto el foco en diferentes aspectos intra e interpersonales. De esta forma se ha considerado como:

- Conocimiento, competencia mental, ejecución o rendimiento. Eficacia en la resolución de problemas, rapidez de procesamiento mental, razonamiento, conjunto de aptitudes, capacidad para aprender, eficacia adquirida.

- Genéticamente determinada o producto del ambiente (resultado del aprendizaje).

- Además, implica aspectos cognitivos, aspectos motivacionales, aspectos personales.

Con estas consideraciones, es lógico pensar que existirá un gran número de modelos y teorías que intentarán definir las AACCII según las características que en ellos prevalecen (Reyzábal, 2002). Así, podemos señalar los principales modelos en el Cuadro 1.

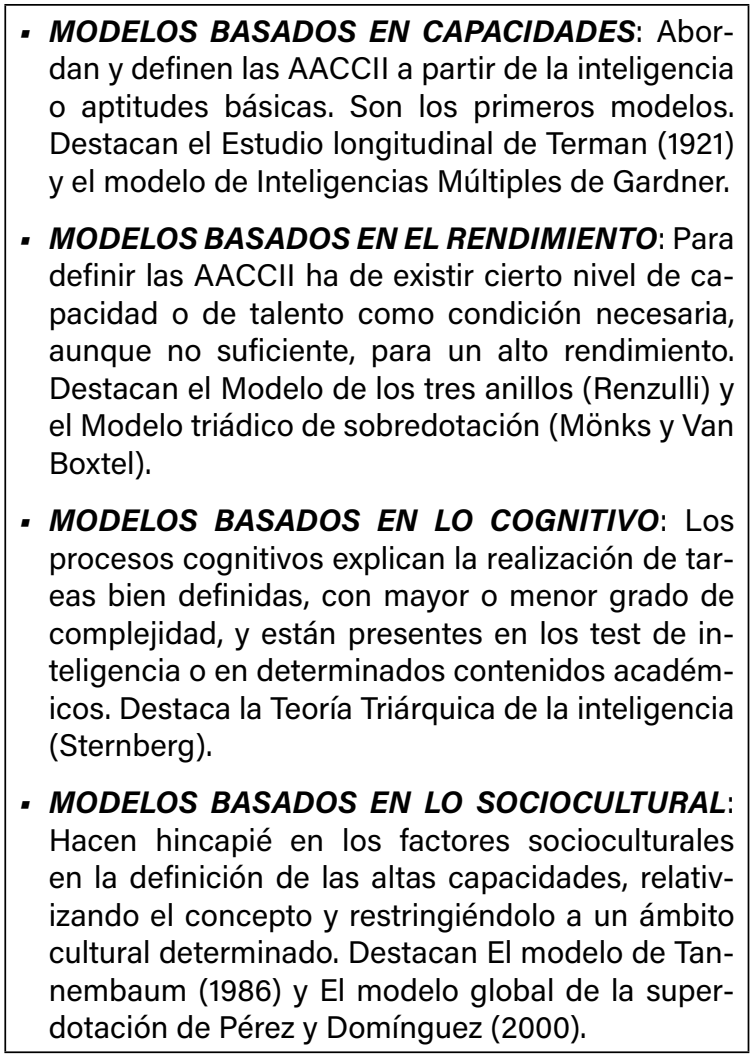

Cuadro 1. Modelos conceptuales generales.

Por lo tanto, como se ha señalado (Luque, 2015), el término y concepto de altas capacidades intelectuales no debe ser una marca clasificadora, estanca o paralizante ni homogénea, sino un elemento o aspecto más de las características individuales y sociales, aunque sea significativo. En este sentido, dentro de un marco inclusivo, se aboga por un modelo amplio en el ámbito de las AACCII en el que se reconocen tanto las capacidades y talentos como el desarrollo de sus competencias, actitudes y valores. Con ello se perseguiría una práctica educativa no solo igualitaria, sino equitativa, en la cual el esfuerzo, la motivación y el interés en los procesos de enseñanza-aprendizaje favorezcan el desarrollo del talento. De esta manera, se buscaría una escuela más capaz para todos, trabajando en la educación de los más capaces.

Entonces, puede considerarse que en la definición, concepto e intervención educativa del alumnado con AACCII tanto el sistema educativo español como el de Andalucía (a través de su Plan de Actuación para la Atención Educativa al Alumnado con Necesidades Específicas de Apoyo Educativo, por presentar Altas Capacidades Intelectuales) seguirían un modelo de capacidades en el que, de acuerdo a Castelló y Batlle (1998), pueden diferenciarse los siguientes perfiles:

- Sobredotación intelectual: Alumnado con un nivel elevado de capacidades cognitivas y aptitudes intelectuales (por encima del percentil 75). Además, este perfil aptitudinal debe ir acompañado de una alta creatividad, por encima del percentil 75.

- Talento simple: Alumnado con una elevada aptitud o competencia en un ámbito específico (por encima del percentil 95) como el verbal, matemático, lógico o creativo.

- Talento complejo: Alumnado con una combinación de varias aptitudes que da lugar a talentos complejos (percentil superior a 80 en, al menos, tres capacidades).

- Talento conglomerado: Alumnado en el que podemos encontrar la combinación de la configuración intelectual del talento complejo y simple.

Cada uno de estos perfiles viene definido por los resultados que este alumnado obtiene en los distintos macroprocesos establecidos en la evaluación de la inteligencia (razonamiento lógico, gestión perceptual, gestión de memoria, razonamiento verbal, razonamiento matemático, aptitud espacial) y la creatividad.

En esos perfiles, teniendo en cuenta estudios y modelos de diversos autores (Castelló \& Batlle 1998; Fernández \& Perales, 2013; Fernández \& Sánchez, 2010; Prieto, Sánchez \& Garrido, 2008; Renzulli, 1977, 1999), pueden apreciarse algunas características generales en el alumnado con AACCII (cuadro 2).

\section{Algunas consideraciones para una evaluación psi- coeducativa}

Como ya hemos señalado, no existen características del comportamiento o de personalidad que sean típicas o asociadas unívocamente a las personas con AACCII. Es por ello que se hace necesario acudir a 
otros aspectos y, más aún, a la integración de factores personales, sociales y de historia familiar, además de los cognitivos para poder explicar el desarrollo personal de cada alumno. En este sentido, sin entrar en debate alguno y aceptando la complejidad del tema de la identificación del alumnado con altas capacidades, su detección es siempre conveniente porque (tratando de evitar el no deseable "etiquetaje" o estigma) constituye un indicador de que la respuesta educativa se adecuará a sus necesidades específicas. Consecuentemente, será necesario el conocimiento de las características individuales y sus potencialidades para el establecimiento de la respuesta educativa. En este apartado, el profesorado, madres y padres tienen una especial actuación en la detección, la cual da paso a las evaluaciones psicoeducativa y pedagógica que terminan por aportar la valoración diagnóstica y el consiguiente establecimiento de una respuesta educativa.

- Elevada capacidad de procesar la información, con gran facilidad para memorizar todo tipo de datos.

- Mayor capacidad de abstracción, razonamiento y generalización, que la que le corresponde a su edad.

- Aprendizaje de conceptos abstractos con capacidad de aplicación de forma práctica.

- Gran curiosidad que les permite aprender más rápidamente.

- Alta motivación intrínseca.

- Independientes. Capacidad superior de autocrítica.

- Nivel elevado de autoestima y autoconfianza.

- Suelen tener ambiciones e ideales muy elevados.

- Se muestran muy sensibles, con un sentido ético muy desarrollado.

- Intereses y preocupaciones profundas sobre el mundo que les rodea.

- Poseen un amplio y complejo vocabulario.

- Aprenden rápidamente a leer y muestran interés por la lectura.

- Expresan sus ideas con claridad. Su diálogo es fluido y rápido con gran comprensión.

- Prefieren el lenguaje oral al escrito como medio de expresión.

- Generación de diferentes soluciones o vías de resolución de un mismo problema.

- Son capaces de crear ideas originales y experimentar cosas nuevas. Tendencia a buscar nuevas alternativas.

- Facilidad para manipular ideas u objetos y obtener nuevas combinaciones.

- Prefieren las tareas complejas a las sencillas.

Cuadro 2. Características generales en el alumnado con altas capacidades intelectuales.

De acuerdo con Richert (1991), podemos hacer algunas observaciones para que las prácticas de identificación sean equitativas y generalizables (cuadro 3)

- Adoptar una definición de sobredotación plural, dando más importancia a la potencialidad y a la valoración de las capacidades, que al simple diagnóstico.

- Cualquier proceso de identificación ha de buscar el desarrollo del potencial del alumno y no alimentar expectativas.

- Hacer uso de datos de capacidades cognitivas, afectivas, de rendimiento, de personalidad, desde diversas fuentes y sin exclusividad en el rendimiento escolar.

- Utilizar adecuadamente los datos de tests de rendimiento, evitando sesgos sobre grupos en desventaja social y cultural.

- Tratar de identificar un $25 \%$ de sujetos, haciendo así que los errores se produzcan más por inclusión que por exclusión.

- Desarrollar programas múltiples con fines de atención a las necesidades de una población diversa en altas capacidades.

- Facilitar la formación del profesorado.

Cuadro 3. Observaciones en la identificación del alumnado con altas capacidades.

Como afirman Arocas, Martínez y Regadera (2002), las estrategias y procedimientos de identificación pueden basarse en medidas formales (calificaciones escolares, tests de rendimiento, pruebas psicométricas, intereses, etc.) informales o subjetivas (cuestionarios, autobiografías, entre otros) y en métodos mixtos. En lo concreto de la evaluación psicopedagógica, deberá seguirse un planteamiento y estructura organizada, así como una planificación ajustada al alumnado y contextos (Artiles \& Jiménez, 2005; Artiles et al., 2003; Comes, Díaz, Luque \& Moliner, 2008; García et al., 2010; Quintero \& Morón, 2011).

Para el caso que se comenta, dentro del Plan de Detección de Alumnado con Altas Capacidades Intelectuales de la Comunidad Autónoma de Andalucía, el proceso se inicia con la aplicación y posterior conjunción de cuestionarios de profesores, padres y madres, continuando con la posterior intervención profesional de los psicólogos educativos y pedagogos (orientadores y orientadoras). Con todo, se considera en sus protocolos:

- Un enfoque en las áreas de capacidades de aprendizaje, creatividad, capacidad de adaptación y competencia social, lenguaje y comunicación.

- Como fuentes de información el profesorado, las familias y los alumnos y alumnas, a través de cuestionarios (padres y madres, profesorado) y nominación entre iguales.

- Son cuestionarios que sirven de base para una eva- 
luación psicoeducativa exhaustiva del alumnado, que determinará su valoración diagnóstica y sus programas de intervención.

- Una evaluación psicopedagógica (llevada a cabo por los profesionales de la psicología, pedagogía o psicopedagogía), dentro de una estructura organizada de estudio y valoración diagnóstica, a la vez que unos planteamientos en el diseño y planificación de la intervención y desarrollo educativo.

Finalmente, se aprecia que la evaluación psicopedagógica constituye una labor interdisciplinar y que, en consecuencia, incorpora la participación de los profesionales que intervienen directamente en los procesos de enseñanza y aprendizaje (profesores, madres y padres). Desde esta coordinación se hace obvio comentar que la evaluación psicopedagógica de los estudiantes con AACCII, puede y debe utilizar procedimientos de identificación estandarizados y no estandarizados para la recogida de información; de forma que se permita la valoración de aspectos como el razonamiento lógico-matemático, memoria, atención, percepción, lenguaje o cálculo. Ya que no se busca un desarrollo instruccional o de docencia exclusivamente, sino la mejora estratégica de las posibilidades cognitivas que, en general, tienen para la adquisición y desarrollo del conocimiento.

\section{Análisis de caso}

De acuerdo a las consideraciones anteriores, siendo muchos los aspectos de interés que podrían analizarse, en este trabajo nos centraremos exclusivamente en aspectos de la evaluación de la capacidad intelectual, su perfil cognitivo, así como, de forma más concisa, en el análisis de la creatividad y de sus aspectos relacionales con el rendimiento académico. Desde ese perfil, haremos una evaluación operativa de la inteligencia, entendida como una facultad general o factor $G$, a la vez que un elenco de factores, aptitudes y otras capacidades que se integran y se relacionan en, dependencia jerárquica de aquel. Para ello, se tomarán principalmente la WISC-IV y el BADYG-E1 como herramientas de evaluación que facilitarán la detección de necesidades y la organización de la respuesta educativa de este alumnado. Así, estimamos una valoración de la inteligencia general y de los factores o aspectos como el razonamiento lógico-matemático, memoria, atención, percepción, lenguaje, cálculo, etc., que en parte la definen.

\section{Datos generales, referencia y objetivos}

Se trata de un alumno de 6 años y 8 meses (al que llamaremos Mario) escolarizado en $1^{\circ}$ de educación primaria, en un colegio público de la provincia de Málaga. De acuerdo con el equipo educativo, y en aplicación del Plan de Detección del Alumnado con Altas Capacidades Intelectuales, se establece un procedimiento de evaluación y la valoración que corresponda, buscando servir de referencia para la acción tutorial, en el ajuste de la respuesta educativa más adecuada. Desde ese momento se mantiene el contacto oportuno con la tutoría y la familia para el asesoramiento y seguimiento psicopedagógicos.

\section{Aspectos evolutivos}

Mario es el mayor de dos hermanos, nacido de embarazo, parto y aspectos de neonatología normales. Desarrollo del lenguaje y motor precoz. Sin historial médico significativo. Estructura y relaciones familiares en un adecuado equilibrio entre la ocupación y la preocupación por el hijo.

\section{Aspectos de historia personal, escolar}

Un resumen de su actividad y rendimiento escolar, extractado del informe de la profesora-tutora, nos aporta los datos recogidos en el cuadro 4:

\section{Aspectos de la exploración psicopedagógica}

\footnotetext{
1. Historia escolar.

Ha cursado Educación Infantil de manera brillante, siendo un alumno que destacaba en todas los ámbitos. Desde su incorporación al sistema educativo ha mostrado producciones muy ricas, en cuanto a cantidad y calidad en las distintas áreas del desarrollo. Asiste con regularidad al centro, con mantenimiento de una conducta de aceptación de normas.

\section{Aspectos afectivos y de rendimiento.}

Su rendimiento está muy por encima de la edad y nivel del aula donde está escolarizado. Tiene un gran nivel de atención, aunque en ocasiones parezca que no está presentando atención al trabajo del aula, siempre responde cuando se le pregunta. Muestra interés hacia las tareas que señala la profesora, si bien, las termina muy rápido y suele demandar más actividad. Hace preguntas inusuales para su edad, en distintas áreas y sobre una gran diversidad de temas.

Trabaja con mucha rapidez y autonomía en la realización de trabajos de clase. Tiene una alta motivación, y manifiesta una gran curiosidad intelectual. Es muy creativo, en especial en aspectos lingüísticos y musicales. Es aceptado por sus compañeros, aunque en ocasiones tiene tendencia a estar aislado, debido a sus áreas de interés y aficiones. Tendencia a relacionarse con adultos.
} 


\begin{abstract}
3. Competencia Curricular.
La profesora fija un nivel aproximado de competencia curricular situado en $2^{\circ}-3^{\circ}$ de EP, añadiendo en aspectos concretos curriculares:

- Un lenguaje oral de articulación correcta, alta riqueza de vocabulario, de diálogo muy fluido y con expresiones muy ricas en detalles. Con gran conocimiento de su entorno próximo. Expresa sus ideas con claridad. El buen dominio que tiene de los instrumentos lingüísticos, favorece su rendimiento académico.

- Lectura muy fluida, con alto nivel de comprensión. Es capaz de leer y recordar gran número de datos.

- En su escritura muestra adecuada coordinación óculo-manual, con sujeción adecuada de lápiz, presión y regularidad en el trazo. Copia normal; dictado de textos y escritura libre con frases e historias cortas. La Profesora expresa que ha alcanzado los objetivos de Lengua Española de $2^{\circ}$ de Educación Primaria. Buen nivel de ortografía.

- En matemáticas hace seriaciones gráficas, conocimiento adecuado de formas básicas, colores, conceptos temporales. Cálculo y operatoria en general, adquiridos.
\end{abstract}

\title{
Cuadro 4 (continuación). Informe Tutorial
}

Dadas las circunstancias personales y de historia escolar de Mario, se ha decidido el siguiente procedimiento de valoración. (Cuadro 5)

\begin{tabular}{l}
\hline Elementos de exploración psiconeurológica. \\
Desarrollo Psicomotor. \\
Organización Perceptiva. \\
Cuestionario de detección de AACC realizado por la \\
tutora. \\
Cuestionario de detección de AACC realizado por la \\
familia. \\
Test de matrices progresivas de Raven Color. \\
Test breve de Inteligencia de Kaufman (K- BIT). \\
Escala de inteligencia de Weschler para niños \\
(WISC-IV). \\
Batería de Aptitudes Diferenciales yenerales \\
(BADyG - E1). \\
Adaptación del test de Pensamiento Creativo de Tor- \\
rance (Expresión figurada). \\
Revisión de materiales escolares. \\
Test de Análisis de la Lectura y Escritura (TALE). \\
Fábulas de L. Düss. \\
Test de la Familia. \\
Entrevistas con la Profesora Tutora del presente cur- \\
so y de la etapa de infantil. \\
Entrevista familiar.
\end{tabular}

Cuadro 5. Técnicas y Procedimientos aplicados

Se opta por la evaluación del alumno con entrevistas de asesoramiento a la profesora -tutora del nivel actual y de la etapa de infantil, prosiguiéndose con la valoración del niño y terminándose con entrevista evolutiva y de asesoramiento a la familia. Seguimiento con el equipo educativo y familia.

\section{Integración de los resultados}

Durante el período de exploración, la actitud del alumno ha sido positiva, de trato afable y de buena interacción con el examinador. Mantiene una conducta de acercamiento a las tareas entre intuitiva y reflexiva, dispuesto y colaborador.
Aspectos de desarrollo: Son adecuados a edad y nivel los aspectos de desarrollo psicomotor. Lateralidad de esquema diestro afirmado. Desarrollo lingüístico por encima de lo esperado a su edad.

\section{Capacidad Intelectual}

Funcionamiento cognitivo: Un análisis global de su inteligencia, valorando los aspectos estructurales y su potencialidad (Raven, K-BIT, WISC IV) revela una capacidad situada en torno a los valores siguientes (Tabla 1). Se aprecia una consistencia entre las pruebas normalizadas de evaluación de su inteligencia, sus valores serían indicadores de una estructura y funcionamiento cognitivo en el extremo superior de la curva normal. Ello dado que:

- Su Cl Total es de 154, situándose en el intervalo de 144-158 (95\%) calculado a partir del Índice de Capacidad General (ICG), al encontrarse una diferencia de más de 23 puntos entre Comprensión Verbal (CV) y Memoria de Trabajo (MT). Los valores que más destacan son CV y Razonamiento Perceptivo (RP).

- Nivel de CV levemente superior a RP que, aunque ambos en unos niveles del percentil 99, expresarían la facilidad del alumno, en lo relativo a aspectos de su escolaridad.

- El soporte que los índices de Memoria de Trabajo y de Velocidad de Procesamiento tienen en esta estructura intelectual indicaría una gran facilidad para el mantenimiento de la información y la conexión de estos conocimientos con otros, facilitando la generalización de los aprendizajes.

Los resultados del WISC-IV analizados con relación a los macroprocesos se resumen en la tabla 2. Estos nos mostrarían que Mario, presenta una puntuación superior al percentil 80 en todos los macroprocesos, lo que sería indicativo de AACCII.

Rendimiento y aptitudes intelectuales: Un análisis global de los resultados obtenidos por Mario en la prueba 
Tabla 1. Funcionamiento cognitivo.

\begin{tabular}{|c|c|c|c|c|}
\hline \multicolumn{5}{|c|}{ Prueba de Raven (Escala Color). } \\
\hline PD. & $\bar{Z}$ & $T$ & PC. & C.I (Equival). \\
\hline 27 & $+2,23$ & 72 & 98.8 & $133(\mathrm{I})$ \\
\hline \multicolumn{5}{|c|}{ Test Breve de Inteligencia de Kaufman (K-BIT) } \\
\hline ÍNDICES & PT & $\mathrm{PC}$ & Categ. Descrip. & Intervalo $95 \%$ \\
\hline Vocabulario & 128 & 97 & Muy Alto & $141-115$ \\
\hline Matrices & 130 & 98 & Muy Alto & $141-119$ \\
\hline Cl Compuesto & 131 & 98 & Muy Alto & $141-121$ \\
\hline \multicolumn{5}{|c|}{ WISC-IV } \\
\hline ÍNDICES & Suma de PE & Punt. Compuesta & Percentil & Intervalo $95 \%$ \\
\hline Comprensión Verbal & 57 & 155 & 99,9 & $143-158$ \\
\hline Razonamiento Perceptivo & 50 & 142 & 99,7 & $130-146$ \\
\hline Memoria de Trabajo & 30 & 127 & 97 & $115-133$ \\
\hline Velocidad de Procesamiento & 31 & 130 & 98 & $116-135$ \\
\hline CI TOTAL (a partir ICG) & $\begin{array}{l}297 \text { (Suma esca- } \\
\text { lares CV+RP) }\end{array}$ & 154 & 99,9 & $144-158$ \\
\hline
\end{tabular}

Fuente: Elaboración propia.

de rendimiento BADYG-E1 y su relación con los macroprocesos, revela una capacidad situada en torno a los valores siguientes (Tabla 3). Se aprecia un funcionamiento alto y consistente en las distintas aptitudes y macroprocesos en el extremo superior de la curva normal.

Los resultados comparados del WISC IV y el BADYG E1 muestran que el alumno se sitúa por encima del percentil 80 en todos los macroprocesos (tabla 4), valorándose un rendimiento intelectual muy alto. En conclusión, en su valoración de capacidad intelectual Mario obtiene, en ese nivel de confianza, una Capacidad Intelectual en la categoría de MUY ALTA.

Creatividad: De acuerdo a la Adaptación del test de Pensamiento Creativo de Torrance (Expresión figurada), los resultados muestran que el alumno presenta un percentil de 85, en sus apartados de originalidad, fluidez, elaboración y flexibilidad. Además, la familia ha aportado trabajos de la etapa de infantil y creaciones del alumno muy ricas en detalles, así como la facilidad del alumno para la música.

Habilidades de Socialización y Conducta.

- Habilidades conceptuales (habilidades cognitivas y de comunicación-académicas) por encima del marco de edad y nivel.

- Habilidades de conducta social: Posee capacidades propias de sus iguales en el establecimiento de relaciones interpersonales, es responsable, sigue reglas y es obedientes a leyes. No obstante, será oportuno incidir en su autoestima y mejora del auto-concepto, así como en habilidades de interacción social.

\section{Aspectos curriculares}

Estilo de aprendizaje: Trabaja individualmente, por pa-
Tabla 2. Análisis de macroprocesos.

\begin{tabular}{|c|c|c|c|}
\hline $\begin{array}{l}\text { Subpruebas y Macropro- } \\
\text { cesos WISC-IV }\end{array}$ & P. E. & $\begin{array}{l}\text { Percen- } \\
\text { til }\end{array}$ & Media PC \\
\hline \multicolumn{4}{|l|}{ 1. RL -Razonamiento lógico } \\
\hline 1.1. Matrices & 18 & 99 & \multirow{3}{*}{99} \\
\hline 1.2. Conceptos & 17 & 99 & \\
\hline 1.3. Semejanzas & 19 & 99 & \\
\hline \multicolumn{4}{|l|}{ 2. GP - Gestión perceptual } \\
\hline 2.1. Matrices & 18 & 99 & \multirow{5}{*}{97} \\
\hline 2.2. Claves & 14 & 91 & \\
\hline 2.3. Conceptos & 17 & 99 & \\
\hline 2.4. Búsqueda de Símbolos & 16 & 98 & \\
\hline 2.5. Figuras Incompletas & 16 & 98 & \\
\hline \multicolumn{4}{|l|}{ 3. GM - Gestión de memoria } \\
\hline 3.1. Dígitos & 16 & 98 & \multirow{3}{*}{95} \\
\hline 3.2. Letras y Números & 14 & 91 & \\
\hline 3.3. Aritmética & 15 & 95 & \\
\hline \multicolumn{4}{|l|}{ 4. RV -Razonamiento verbal } \\
\hline 4.1. Información & 17 & 99 & \multirow{5}{*}{99} \\
\hline 4.2. Adivinanzas & 15 & 91 & \\
\hline 4.3. Vocabulario & 19 & 99 & \\
\hline 4.4. Comprensión & 19 & 99 & \\
\hline 4.5. Semejanzas & 19 & 99 & \\
\hline \multicolumn{4}{|c|}{ 5. RM -Razonamiento matemático } \\
\hline 5.1. Aritmética & 15 & 95 & 89 \\
\hline \multicolumn{4}{|l|}{ 6. AE -Aptitud espacial } \\
\hline 6.1. Cubos & 15 & 95 & \multirow{2}{*}{97} \\
\hline 6.2. Figuras Incompletas & 16 & 98 & \\
\hline
\end{tabular}

Fuente: Elaboración propia. 
Tabla 3. BADYG-E1 y Macroprocesos.

\begin{tabular}{|c|c|c|c|}
\hline $\begin{array}{l}\text { Aptitudes y Macropro- } \\
\text { cesos }\end{array}$ & PD & Porcentil & Media PC \\
\hline \multicolumn{4}{|c|}{ 1. $R L$-Razonamiento lógico } \\
\hline 1.1. Matrices lógicas (Re) & 16 & 99 & \multirow{3}{*}{90} \\
\hline $\begin{array}{l}\text { 1.2. Relaciones analógi- } \\
\text { cas (Rv) }\end{array}$ & 12 & 85 & \\
\hline $\begin{array}{l}\text { 1.3. Problemas numéri- } \\
\text { cos-verbales (Rn) }\end{array}$ & 13 & 85 & \\
\hline \multicolumn{4}{|l|}{ 2. GP - Gestión perceptual } \\
\hline $\begin{array}{l}\text { 2.1. Discriminación de } \\
\text { diferencias (De) }\end{array}$ & 17 & 99 & \multirow[t]{2}{*}{88} \\
\hline 2.2. Figuras giradas $(\mathrm{Se})$ & 14 & 77 & \\
\hline \multicolumn{4}{|c|}{ 3. GM - Gestión de memoria } \\
\hline Memoria inmediata (Ma) & 25 & 95 & 95 \\
\hline \multicolumn{4}{|c|}{ 4. RV -Razonamiento verbal } \\
\hline $\begin{array}{l}\text { 4.1. Problemas numéri- } \\
\text { cos-verbales }(R n)\end{array}$ & 13 & 85 & \multirow{2}{*}{92} \\
\hline $\begin{array}{l}\text { 4.2. Órdenes verbales } \\
\text { complejas (Sv) }\end{array}$ & 15 & 98 & \\
\hline \multicolumn{4}{|c|}{ 5. RM -Razonamiento matemático } \\
\hline $\begin{array}{l}\text { 5.1. Problemas numéri- } \\
\text { cos-verbales (Rn) }\end{array}$ & 14 & 89 & \multirow{2}{*}{92} \\
\hline $\begin{array}{l}\text { 5.2. Cálculo numérico } \\
\text { (Sn) }\end{array}$ & 15 & 95 & \\
\hline \multicolumn{4}{|l|}{ 6. AE -Aptitud espacial } \\
\hline 6.1. Figuras giradas (Se) & 17 & 95 & \\
\hline $\begin{array}{l}\text { 6.2. Discriminación de } \\
\text { diferencias }(\mathrm{De})\end{array}$ & 14 & 87 & 91 \\
\hline
\end{tabular}

rejas y en grupo, con una alta autonomía. No es impulsivo, siendo sensible a las reprimendas y, en ocasiones, intentándolas justificar y razonar. Trabaja con rapidez, de gran constancia y no se desanima fácilmente.

Aprendizaje de la lectura: Adecuado conocimiento y discriminación de letras, sílabas y palabras. Lectura de textos sin errores, aunque en alguna ocasión hace rectificaciones. A partir de los resultados de las cuatro lecturas de la prueba, se obtiene una velocidad lectora media en el intervalo de 90-100 ppm. Comprensión lectora, en porcentajes altos.

Aprendizaje de la escritura: Su escritura es de caracteres ligados, con tamaño de la letra normalizado, grafía algo irregular, con alguna oscilación, rectas y curvas adecuadas. Adaptación a la pauta. Escritura de dictado y copia sin errores de ortografía normativa.

Aprendizaje matemático: Buen nivel de razonamiento y de resolución de problemas. Realiza operaciones básicas de suma, resta, multiplicación y se está iniciando en la división.

En una valoración de este tipo de aprendizajes, se puede concluir la necesidad de seguir favoreciendo la comprensión lectora, la expresión escrita y el aprendizaje matemático, así como el acceso a la información de manera autónoma.

\section{Personalidad y Conducta}

Dado el carácter reservado de este tipo de información, se mantendrá el contacto oportuno con la familia, profesorado y alumno, transmitiendo las orientaciones oportunas. No obstante, no se aprecian datos de relevancia con influencia en su aprendizaje y rendimiento académico.

\section{Resumen y conclusiones}

De acuerdo a lo que antecede y para este particular momento evolutivo de Mario, puede precisarse lo siguiente:

- Capacidad intelectual muy alta.

- Capacidad de adaptación y de habilidades sociales normal respecto a sus iguales.

- Amplio y complejo vocabulario con un diálogo fluido y rápido.

- Interés y facilidad hacia la lectura.

- Capacidad para crear ideas originales.

- Generación de diferentes soluciones o vías de resolución de un mismo problema.

- Buen nivel de conducta adaptativa y habilidades sociales.

- Facilidad para los aspectos musicales.

En un resumen de valoración diagnóstica, considerando sus aspectos evolutivos y de conducta personal y social, sin perjuicio de lo que aporten posteriores valoraciones, y como una base de partida para el seguimiento y evaluación psicológicos y psicopedagógicos, su caso puede incluirse en el Censo de Necesidades Específicas de Apoyo Educativo por Altas Capacidades Intelectuales (sobredotación intelectual).

Tabla 4. Macroprocesos

\begin{tabular}{lllllll}
\hline & $\begin{array}{l}\text { Raz. lógico } \\
(\mathbf{R L})\end{array}$ & $\begin{array}{l}\text { Raz. verbal } \\
(\mathbf{R V})\end{array}$ & $\begin{array}{l}\text { Raz. matemáti- } \\
\text { co (RM) }\end{array}$ & $\begin{array}{l}\text { Gestión Per- } \\
\text { ceptual (GP) }\end{array}$ & $\begin{array}{l}\text { Gestión de } \\
\text { Memoria (GM) }\end{array}$ & $\begin{array}{l}\text { Aptitud Espa- } \\
\text { cial (AE) }\end{array}$ \\
\hline WISC IV & 99 & 99 & 95 & 98 & 89 & 97 \\
BADYG E1 & 90 & 92 & 92 & 88 & 95 & 91 \\
\hline
\end{tabular}

Fuente: Elaboración propia. 
Diagnóstico con el que se sugiere el desarrollo de una adaptación curricular de ampliación, que permita adquirir los conocimientos del nivel superior donde el alumno está escolarizado, y en último término, valorar la posibilidad de flexibilización del período de escolarización del alumno. Se sugiere la aplicación de un programa de enriquecimiento en el desarrollo de estrategias de trabajo intelectual y de la creatividad, así como la observación en la esfera de desarrollo personal y de habilidades sociales. Esto con el fin de seguir trabajando la relación con sus iguales, previniendo dificultades en la esfera socio-emocional.

En función de esa valoración y dentro de un marco educativo ordinario, se tratará de adecuar el currículum a las necesidades educativas que Mario presenta en una escolarización en centro y grupo ordinario a tiempo completo, con atención del psicólogo, pedagogo o psicopedagogo, profesorado especialista en AACCII (si en el centro hay la formación), así como del profesor-tutor y del conjunto del equipo educativo.

\section{Orientación y Consejo psicoeducativo}

Dado que las necesidades educativas que Mario presenta se derivan de sus características de personalidad y de desarrollo, la respuesta educativa debe adoptar como marco de intervención la facilitación de su desarrollo individual y social, desarrollo de capacidades en general y de los aspectos académicos, de tal forma que sirvan de instrumentos para la orientación en su vida.

Desde este marco, contando con que su intervención escolar podrá precisar de un currículum adecuado a sus necesidades, se debería reflexionar sobre el desarrollo de su escolaridad de acuerdo a los siguientes puntos.

\section{Orientaciones generales}

1. Respecto a la familia, incrementar la relación con el centro y tutoría, así como mantener el grado de coordinación adecuado, a fin de conseguir los beneficios de toda acción conjunta.

2. Continuar la acción tutorial con el alumno para que, acercándose a su individualidad, permita su mejor conocimiento y se consiga adecuar el currículum ordinario a sus necesidades educativas. En este punto apreciamos positivamente la atención del equipo educativo para mejorar el desarrollo académico del niño. Deberían plantearse, desde este punto de vista, actividades variadas, con un grado de complejidad cada vez mayor, y favorecer la coordinación entre los distintos profesionales que se encarguen de la atención del alumno. En este sentido, la reflexión sobre la práctica docente podría enfocarse en:

- Actividades que permitan el trabajo autónomo y combinen distintas áreas o mate- rias, a la vez que se fomenten actividades de trabajo en grupo, donde el alumno asuma la tarea de coordinador de situaciones de trabajo.

- Enfatizar la comprensión más que la memorización, recordando que, a menudo, se apartará de los objetivos establecidos.

- Desarrollar prácticas docentes sobre un aprendizaje en el alumnado de AACCII que facilite su autonomía, la comunicación y el acceso a la información, etc.

- Realizar un cambio de metodologías y de evaluación, creando espacios y tiempos diferenciados, fomentando el trabajo a partir de distintas agrupaciones, generando actividades diferentes, favoreciendo la creatividad y el pensamiento divergente.

3. En la intervención psicopedagógica concreta del alumno, será conveniente el trabajo en:

- Aspectos curriculares: elaboración de una adaptación curricular de ampliación con objetivos de segundo de educación primaria.

- Estrategias de trabajo intelectual.

- Desarrollar un programa de enriquecimiento.

- Fomentar la creatividad.

- Desarrollo personal y de habilidades sociales.

\section{Algunas conclusiones para la reflexión}

El alumno en cuestión presenta altos niveles de capacidad intelectual, de creatividad y de motivación hacia las tareas, buen nivel lectoescritor y de razonamiento lógico-matemático, habilidades sociales y de adaptación adecuadas, así como una gran capacidad de memoria, entre otros aspectos. En consecuencia, podría afirmarse que se trata de un caso de AACCII por sobredotación intelectual. En la etapa de infantil, no se realizó una evaluación psicopedagógica, la cual hubiera podido establecer una respuesta ajustada a su persona, facilitando la adecuación curricular, personal y social, habiéndose considerado la flexibilización de su período de escolarización en la incorporación a la educación primaria.

Si bien siempre ha existido cierta controversia sobre si se deben detectar las AACCII en la etapa de educación infantil o más adelante, en la etapa de la educación primaria, en los países donde se lleva más tiempo desarrollando programas de atención a las AACCII, se mantiene la idea de una detección lo más temprana posible (Chamberlin, Buchanan \& Vercimak, 2007) con el fin de ajustar la respuesta educativa y seguir favoreciendo la voluntad e interés en el proceso de aprender, consiguiendo así un mejor progreso en su desarrollo acadé- 
mico, personal y social. No obstante, en nuestro caso, la permanencia con su grupo en Educación Infantil ha servido para la potenciación de sus relaciones sociales, la mejora de sus habilidades para con el grupo, así como un desarrollo normalizado de su autoestima.

Al realizar una valoración de los resultados obtenidos en el WISC- IV y en el BADYG E1, se podría apuntar a una inteligencia cristalizada (capacidad para enfrentarse a tareas de entrenamiento, escolaridad y socialización) levemente mayor a la fluida (capacidad de solución problemas previamente desconocidos, mediante adaptación y flexibilidad), si bien las diferencias no son significativas. A este respecto, puede decirse que el análisis de su capacidad intelectual nos sugiere una estructura cognitiva que se ha ido modelando sobre elementos verbales (CV). Ello, desde la base de una organización perceptivo-atencional muy alta (RP), construcción que es fruto del inicio de los procesos y actividades escolares y de la propia potencialidad del alumno, los cuales le han permitido desarrollar habilidades y estrategias de trabajo intelectual (función ejecutiva y memoria de trabajo).

En nuestro caso se puede observar que, desde la inferencia de los valores obtenidos en el índice MT, el alumno presentaría un nivel muy alto de ejecución en su procesamiento y representación de la información con la que trabaja tanto a nivel de lenguaje como en lo visoperceptivo y en el control ejecutivo de la información. De igual forma, se distingue un excelente rendimiento en los resultados de los subtest, indicando un alto nivel en los componentes atencional y de razonamiento visoperceptivo, así como en un razonamiento lógico y de base lingüística.

Finalmente, los aspectos perceptivo-atencionales que se observan en la VP (percepción y selección visual, coordinación visomotora, flexibilidad cognitiva, atención y motivación) del WISC- IV y en la prueba de atención del Badyg, siendo altos, resultan más bajos respecto a las propias puntuaciones. Se trata de observaciones que se aprecian en otros estudios con estos alumnos (Flanagan \& Kaufman, 2006; Watkins, Greenawalt \& Marcell, 2002). Esto podría ser un indicativo de su rendimiento ante actividades habituales o de carácter rutinario, lo que nos llevaría a replantearnos la metodología de trabajo que realizamos con este alumnado.

Todo ello nos conduce a la conveniencia de proseguir con una intervención, principalmente a tres bandas:

- Estrategias de trabajo intelectual a fin de obtener una mejora de su rendimiento cognitivo.

- Desarrollo académico a través de una adaptación curricular que favorezca el rendimiento escolar.

- Fomento de la creatividad y el pensamiento divergente, y estimulación de sus áreas de intereses.
Finalmente, no debemos olvidar la importancia de las necesidades socio-emocionales de estos alumnos, asumiendo que la valoración del alumno no se sustenta solo sobre el $\mathrm{Cl}$, sino sobre el funcionamiento intelectual conceptual, práctico y social (Luque, 2014), así como en un profundo conocimiento de su desarrollo afectivo-emocional, desarrollo social y moral. Tales aspectos conforman el crecimiento personal del alumnado en su evolución individual y como miembro de su comunidad.

En conjunción con los apartados anteriores y a modo de conclusión, pueden hacerse algunas consideraciones para la reflexión.

Siendo la evaluación e intervención psicoeducativa herramientas de la psicología educacional, cualquier profesional de ésta debe tener en cuenta que el enfoque clínico-médico se sustituye por otro constructivo-educacional. De este modo, se desplaza el acento desde el estudio de casos individuales y la búsqueda de instrumentos de diagnóstico y tratamiento individual a una intervención ecológica centrada en los contextos educativos y sociales como factores causales y objetivos de cambio (Trianes, Luque \& Fernández, 2014). En nuestro caso de análisis, la intervención psicoeducativa debe proporcionar recursos y métodos al profesorado para la mejora del aprendizaje escolar, no solo relativos al dominio de las materias del currículum, sino a la educación de habilidades para la vida, de actitudes y valores morales y democráticos que componen un desarrollo personal integral del alumnado. Así mismo, será necesaria la intervención en los aspectos afectivos del alumno a fin de integrar su desarrollo personal y social. En este sentido, será oportuna la aplicación de programas de inteligencia emocional.

A pesar de que en buena parte de los trabajos y avances en el estudio sobre las AACCII siguen cargándose las tintas sobre los aspectos intelectuales, quizá porque se estimen más accesibles u objetivos en su medición, o bien porque se crea que su valor es básico para explicar otros aspectos, debemos aceptar, por obvio, que el niño o niña con altas capacidades intelectuales es una persona en la que se da una integración de talento, características personales propias y un contexto de desarrollo. En consecuencia, la intervención psicoeducativa en el alumnado con AACCII irá encaminada a describir, explicar y aportar recursos al aprendizaje y logro de objetivos de construcción personal, desarrollo de competencias sociales, emocionales y morales.

Finalmente, desde la visión de necesidades educativas, la atención al alumnado con altas capacidades intelectuales puede aportar al profesorado la suficiente reflexión educadora para valorar su atención a la diversidad como propia de su quehacer docente y tutorial. Ciertamente, podrá necesitar de recursos, apoyos, materiales o actividades, pero todo ello surgirá de manera 
natural del conocimiento del alumno y de su acción tutorial, ya que esta le define como un profesor o profesora implicado y comprometido con el desarrollo personal de sus alumnos. Solo de esta manera podremos estar hablando de inclusión y de un marco favorecedor de éxito y de desarrollo personal y social.

\section{Referencias}

Arocas, E., Martínez, P. \& Regadera, M. (2002). Orientaciones para la Evaluación Psicopedagógica del Alumnado con Altas Capacidades. Valencia: Consellería de Cultura y Educación de la Generalitat Valenciana.

Artiles, C. \& Jiménez, J. (Coord.). (2005). La respuesta educativa para el alumnado con altas capacidades intelectuales. Volumen 3. Las Palmas de Gran Canaria: Universidad de las Palmas de Gran Canaria.

Artiles, C., Jiménez, J., Alonso, P., Guzmán, R., Vicente, L. \& Álvarez, J. (2003). Cómo trabajar en el aula con el alumnado de altas capacidades. Aspectos metodológicos y de evaluación de aprendizajes. En C. Artiles, J. Jiménez, P. Alonso, R. Guzmán, L. Vicente \& J. Álvarez, Programa para la atención educativa al alumnado con altas capacidades de la Comunidad Autónoma de Canarias. Orientaciones para la detección e identificación del alumnado que presenta altas capacidades y su intervención educativa. Guía para profesionales de la educación (86-104). Consejería de Educación del Gobierno de Canarias: Dirección General de Ordenación e Innovación Educativa.

Calero, M., García, M. \& Gómez, M. (2007). El alumnado con sobredotación intelectual. Conceptualización, evaluación y respuesta educativa. Sevilla: Junta de Andalucía.

Castelló, A. \& Batlle, C. (1998). Aspectos teóricos e instrumentales en la identificación del alumno superdotado y talentoso. Propuesta de un protocolo. FAISCA: Revista de Altas Capacidades, 6, 26-66. Recuperado de http://revistas.ucm.es/ index.php/FAIS/article/view/FAIS9898110026A

Chamberlin, S., Buchanan, M. \& Vercimak, D. (2007). Serving twice-exceptional preschoolers: Blending gifted education and early childhood special education practices in assessment and program planning. Journal for the Education of the Gifted, 30(3), 372-394. Recuperado de http:// files.eric.ed.gov/fulltext/EJ756559.pdf

Comes, G., Díaz, E., Luque, A. \& Moliner, O. (2008). La evaluación psicopedagógica del alumnado con altas capacidades intelectuales. Revista Educación Inclusiva, 103. Recuperado de ht- tps://dialnet.unirioja.es/servlet/articulo?codigo $=3011828$

Fernández, C. \& Perales, R. (2013). Los alumnos más capaces en España. Normativa e incidencia en el diagnóstico y la educación/The most capable students in Spain. Normative and incidence in diagnosis and education. REOP-Revista Española de Orientación y Psicopedagogía, 24(1), 7-24. Recuperado de http://revistas.uned.es/ index.php/reop/article/view/11267

Fernández, M. \& Sánchez, M. (2010). Cómo detectar y evaluar a los alumnos con altas capacidades intelectuales: guía para profesores y orientadores. Sevilla: MAD.

Flanagan, D. \& Kaufman, A. (2006). Claves para la Evaluación con el WISC-IV. Madrid: TEA Ediciones.

García, C., Sánchez, M., Fernández, M., Martínez, G., Prieto, M. \& Martín, M. (2010). Modelo de identificación de alumnos con altas habilidades de Educación Secundaria. Revista electrónica interuniversitaria de formación del profesorado, 13(1), 63-74. Recuperado de http:// www.aufop.com/aufop/uploaded files/articulos/1268616568.pdf

Luque, D. (2014). Necesidades Educativas Específicas del alumnado con Altas Capacidades Intelectuales. En Fernández-Molina, M. (Coord.), Título Propio en Altas Capacidades Intelectuales - Material Docente. Málaga: Facultad de Ciencias de la Educación. Universidad de Málaga.

Luque, D. (2015). Intervención Psicopedagógica en el Alumnado con Altas Capacidades Intelectuales: aspectos de continua reflexión. Revista Asociación de Orientadores y Orientadoras de Málaga, 19, 9-17. Recuperado de https://issuu. com/revista aosma/docs/aosma19

Prieto, M., Sánchez, C. \& Garrido, C. (2008). Características del alumnado con altas capacidades intelectuales. En A. Gómez, C. Cerón, R. Lineros \& M. Gómez, Atención a la diversidad. Materiales para la formación del profesorado (1-22). Murcia: Servicio de Atención a la Diversidad.

Quintero, J. \& Morón, A. (2011). Alumnado con Altas Capacidades Intelectuales. Madrid: Cuadernos de Recursos 4.

Renzulli, J. (1977). The enrichment triad model: A guide for developing defensible programs for the gifted and talented. Michigan: Creative Learning Press.

Renzulli, J. (1999). What is this thing called giftedness, and how do we develop it? A twenty - five year perspective. Journal for the Education of the Gif- 
ted, 23(1), 3-54. Recuperado de http://jeg.sagepub.com/content/23/1/3.abstract

Reyzábal, M. (2002). Respuestas educativas al alumnado con altas capacidades intelectuales. Madrid: MEC. Dirección General de Promoción Educativa.

Richert, E. (1991). Rampant problems and promising practices in identification. Gifted Child Quarterly, 31(4), 81-96. Recuperado de http://eric. ed.gov/?id=EJ380191

Torrego, J. (2011). Alumnos con altas capacidades y aprendizaje cooperativo. Madrid: Fundación Pryconsa.

Trianes, M., Luque, D. \& Fernández, F. (2014). Psicología de la Educación para el Grado en Psicología. Sevilla: Universidad de Sevilla.

Watkins, M., Greenawalt, C. \& Marcell, C. (2002). Factor structure of the Wechsler Intelligence Scale for Children-Third Edition among gifted students. Educational and Psychological Measurement, 62(1), 164-172. Recuperado de http://epm.sagepub.com/content/62/1/164.abstract 